Mizuno, T., et al. Generation of Heat and Products During Plasma Electrolysis. in Eleventh International Conference on Condensed Matter Nuclear Science. 2004. Marseille, France.

\title{
Generation of Heat and Products During Plasma Electrolysis
}

\author{
Tadahiko Mizuno, David. Y. Chung ${ }^{(1)}$, F. Sesftel ${ }^{(1)}$ and Yoshiaki Aoki \\ Hokkaido University, Kita-ku Kita-13 Nishi-8, Sapporo 060-8628, Japan \\ (1): Department of Physics, Howard University, Washington, D.C. 20059, USA
}

\begin{abstract}
Direct decomposition of water is very difficult in normal conditions. Hydrogen gas can be usually obtained by electrolysis or by a pyrolysis reaction at high temperatures, starting at approximately $3700^{\circ} \mathrm{C}$. However, as we have already reported, anomalous heat generation can occur during plasma electrolysis, and this process makes it rather easy to achieve both electrolysis and pyrolysis simultaneously. In this paper we describe anomalous amounts of hydrogen and oxygen gas generated during plasma electrolysis. The generation of hydrogen far in excess of amounts predicted by Faraday's law is continuously observed when conditions such as temperature, current density, input voltage and electrode surface are suitable. Non-Faraday generation of hydrogen gas sometimes produces more than 80 times as much hydrogen as normal electrolysis does. Unfortunately there have been few claimed replications of excess hydrogen, even in rare cases in which excess heat is claimed. In most cases, no excess heat or hydrogen is observed. The reaction products found after electrolysis were different after excess heat generation.
\end{abstract}

Key words: plasma electrolysis, pyrolysis, hydrogen generation, transmutation

\section{Introduction}

Hydrogen gas can be easily obtained by electrolysis. However, direct decomposition of water is very difficult in normal conditions. The pyrolysis reaction occurs at high temperatures, starting at approximately $3700^{\circ} \mathrm{C}(1,2)$. We have already reported anomalous heat generation during plasma electrolysis $(3,4)$. Some researchers have attempted to replicate the phenomenon; however, they report difficulty generating a high level of excess heat. They tend to increase input voltage to a very high value, hundreds of volts, a technique we do not recommend. It is very important to replicate the excess heat and other products during plasma electrolysis.

The generation of hydrogen in excess of Faraday's law is continuously observed then conditions such as temperature, current density, input voltage and electrode surface are suitable. Non-Faraday generation of hydrogen gas sometimes produces more than 80 times as much hydrogen as normal electrolysis does. Usually, the plasma state can be easily started if input voltage is increased up to $140 \mathrm{~V}$ at a rather high temperature. ${ }^{(5,6,7)}$ when the plasma forms, a great deal of vapor and hydrogen gas are released from the cell. At the same time, this effluent gas removes heat (enthalpy) which then cannot be detected with calorimetry based only on temperature. It is difficult to calibrate the exact enthalpy balance. A mixture of gas and heat is especially complicated and difficult to measure. In this paper we describe a heat measurement system used to during plasma electrolysis that accounts for all enthalpy. 


\section{Experiment}

\subsection{Electrolysis cell}

Figure 1 shows the experimental set up. We measure many parameters including sample surface temperature, neutron and x-ray emission, mass spectrum of gas, input and output power, and so on. Figure 2 shows the schematic sketch of the cell and measurement system $^{(1-2)}$. The cell is made of the Pyrex glass $10 \mathrm{~cm}$ diameter and $17 \mathrm{~cm}$ in height and $1000 \mathrm{cc}$ in solution capacity. It is closed with a Teflon rubber cap, $7 \mathrm{~cm}$ in diameter. The cap has several holes in it, three for platinum resistance temperature detectors (RTD)

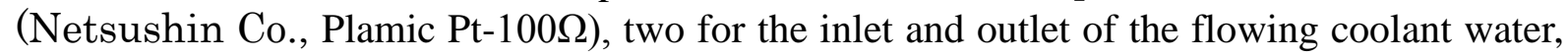
and one to hold a funnel that captures the effluent gas from the cathode. The funnel is made of quartz glass, and is $5 \mathrm{~cm}$ in the diameter at the top of the cell, and $12 \mathrm{~cm}$ in length. Gas leaving the top of the funnel flows into a water-cooled condenser, which is connected to the funnel with another Teflon rubber cap. This is shown in Figure 3 and photo 4.

\subsection{Measurement of hydrogen gas}

A mixture of steam, hydrogen and oxygen (from pyrolysis) passes from the cell to the condenser. The steam condenses and falls back into the cell. An 8-mm diameter Tigon tube is coupled with the gas exit of the condenser, connecting it to a gas flow meter (Kofloc Co., model 3100, controller: Kofloc Co., model CR-700).

The flow to voltage transformer element is a heated tube of thermal flow meter system, the minimum detection rate of hydrogen gas flow is $0.001 \mathrm{cc} / \mathrm{s}$, and the resolution is within $1 \%$. The power output from the measurement system was led to the computer through a logger.

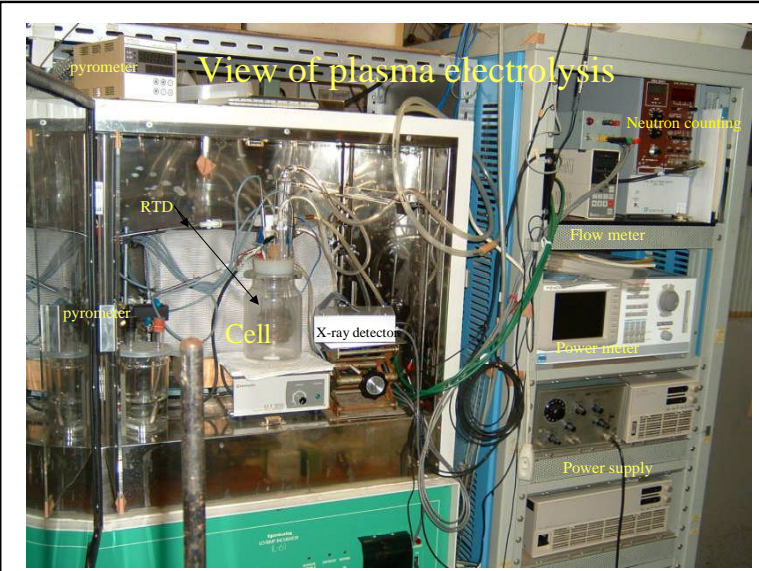

Fig. 1. Photo of the experimental setup

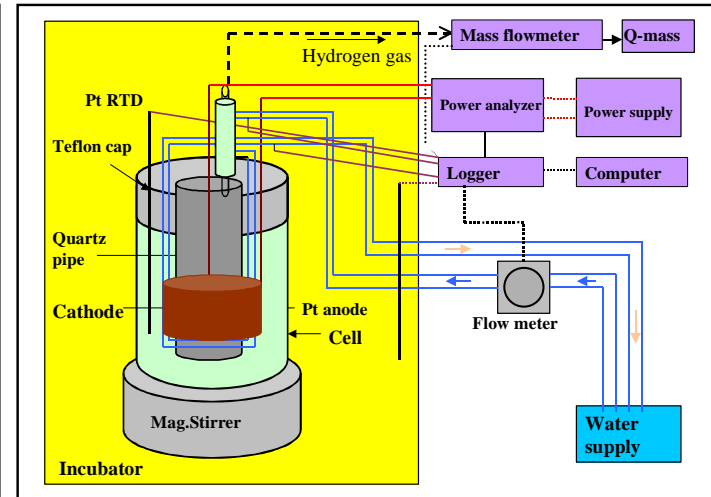

Fig. 2. Sketch of experimental setup 


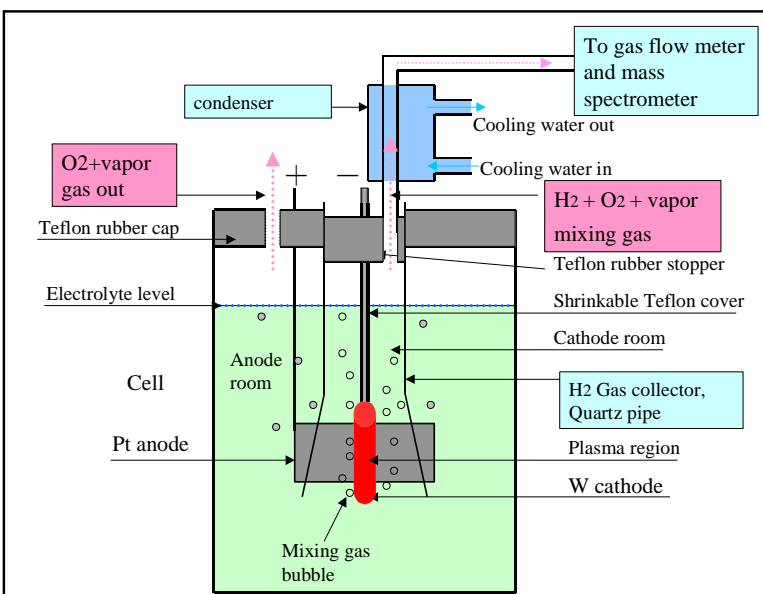

Fig. 3. Detail of the gas measurement

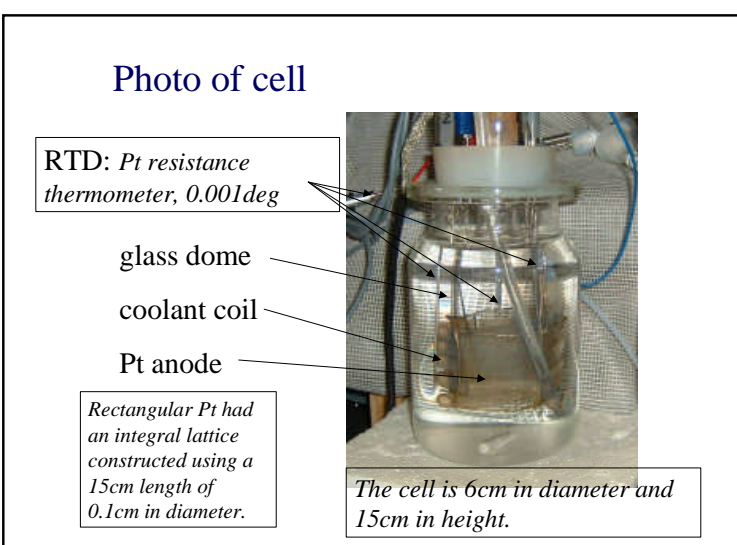

Fig. 4. Photo of cell

After path through the flow meter, the gas goes to a mass spectra analysis system. A small amount of constant volume of the gas such as $0.001 \mathrm{cc} / \mathrm{s}$ paths continuously through a needle valve and was analyzed by a quadruple mass analysis method.

The main composition of gas released from the cathode was then continuously analyzed by above-mentioned method.

\subsection{Calorimetry}

Temperature measurements were made with $1.5 \mathrm{~mm}$ diameter RTDs. Calorimetry was performed by combining the flow and isoperibolic method.

Flow calorimetry is based on the temperature change of the cooling water. The cooling water is tap water flowing through Tigon tubing. It passes first through a constant temperature bath to keep the temperature constant. From there, it flows through the outer jacket of the condenser, and then through the coil of tubing wrapped around the funnel. (The outside of this cooling water coil is covered with the anode, a platinum mesh). The flow rate is measured with a turbine meter (Japan Flow Control Ltd., model T-1965B). The inlet temperature is measured before the cooling water enters the condenser, and outlet temperature is measured where it exits the cell. Heat from both condensation and glow discharge electrolysis are combined together.

Isoperibolic calorimetry is performed by placing three other RTDs were in the cell electrolyte at different depths in the solution to measure the temperature. The solution is mixed with a magnetic stirrer.

Figure 5 shows the notional sketch for heat measurement. Heat out can be divided into several factors. These are energy for water decomposition, heat of electrolyte, heat bring by the coolant, heat releasing from the call wall and heat releasing with the vapor through the cell plug. 
The heat balance is estimated by input and output formulas, input and output power is given in the following equations:

$$
\begin{aligned}
& \cdot \text { Input }(\mathrm{J})=\mathbf{I} \text { (current) } \cdot \mathbf{V}(\text { Volt }) \cdot \mathbf{t} \\
& \cdot \text { Out }=\mathrm{Hg}+\mathrm{Hw}+\mathrm{HC}+\mathrm{Hr}+\mathrm{Hv}
\end{aligned}
$$

here, $\mathrm{Hg}=$ Heat of decomposition $=\int 1.48 \cdot d I \cdot d t$

$$
\mathrm{Hw}=\text { Electrolyte heat }=\int W w \cdot C w \cdot \delta T
$$

- $W w$ :electrolyte weight, $C w$ :heat capacity, $\delta T$ :temperature difference

$$
\mathrm{Hc}=\text { Heat of coolant }=\int W c \cdot C c \cdot \delta T
$$

- $W c$ :coolant weight, $C c$ :heat capacity, $\delta T$ :temperature difference

$$
\cdot \mathrm{Hr}=\text { Heat release }=\int(W w \cdot C w+W c \cdot C c) T r
$$

- $T r$ : temperature change

$\cdot \mathrm{Hv}=$ vapor $=W v \cdot C c$

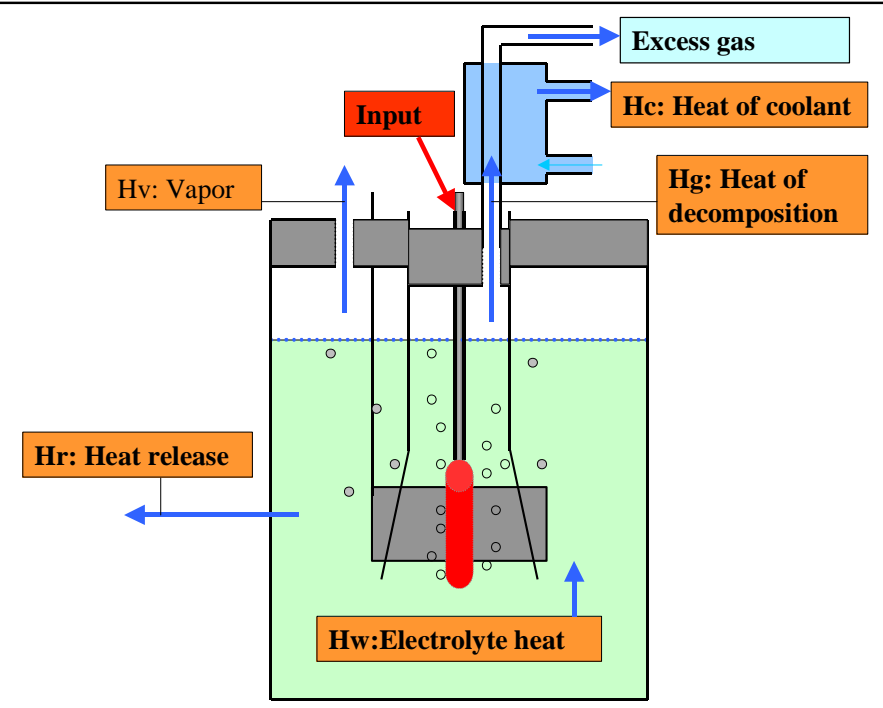

Fig. 5. Schematic representation of heat balance

The heat balance is calculation is straightforward. Input power is only from the electric power source. Output is divided into several parts. The first factor is heat of water decomposition (designated $\mathrm{Hg}$ ). It is easily calculated from the total electric current. The second factor is electrolyte enthalpy $(\mathrm{Hw})$. It is easily derived from the solution temperature difference. The third factor is heat removed by the coolant (Hc). This is measured from the temperature difference between the coolant inlet and outlet, and the coolant flow rate. The fourth factor is heat release from the cell $(\mathrm{Hr})$. This is rather complicated and can be estimated with a semi-empirical equation. The fifth factor is heat release by vapor (Hv). This is difficult to measure precisely. However, I have measured most of the heat in the condenser directly by monitoring the inlet and outlet temperature of the cooling water that passes through the condenser outer jacket.

If there is excess hydrogen and oxygen gas, we have to measure the gas volume precisely, because even a small volume of gas removes a large amount of enthalpy. This is done with the precision gas flow meter. The first factor, water decomposition $(\mathrm{Hg})$ has a large effect on the rest of the equation. 


\subsection{Electrode and solution}

The electrode is tungsten wire, $1.5 \mathrm{~mm}$ in diameter and $15 \mathrm{~cm}$ in length. The upper $13 \mathrm{~cm}$ of the wire is covered with shrink-wrap Teflon and the bottom $2 \mathrm{~cm}$ is exposed to the electrolyte and acts as an electrode. The light water solution was made from high purity $\mathrm{K}_{2} \mathrm{CO}_{3}$ reagent at $0.2 \mathrm{M}$ concentration.
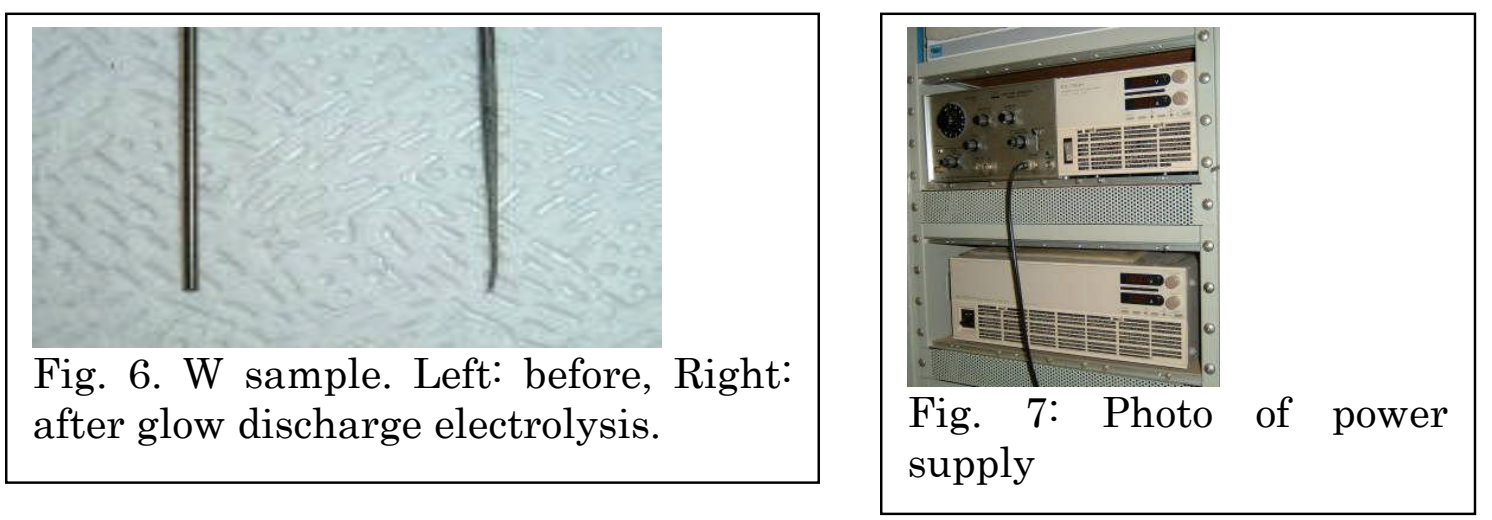

\subsection{Power supply}

Data from the electric power supply (Takasago, model EH1500H) was collected with a power meter (Yokogawa Co., model PZ4000) and averaged every 5 seconds. The sampling time was $40 \mu$ s and the data length was $100 \mathrm{k}$.

\subsection{Data collection}

All data, including the mass of cooling water flow from the flow calorie measurement, the temperature of coolant entrance and exit, electrolyte temperature measured by three RTDs, input voltage, current, electric power and the amount of the hydrogen gas generated were collected by a data logger (Agilent Co., model 34970A), and stored in a personal computer.

\section{7 element analysis}

The sample electrodes and the electrolyte were subjected to element detection by means of energy dispersive X-ray spectroscopy (EDX), Auger electron spectroscopy (AES), secondary ion mass spectroscopy (SIMS) and electron probe micro analyzer (EPMA).

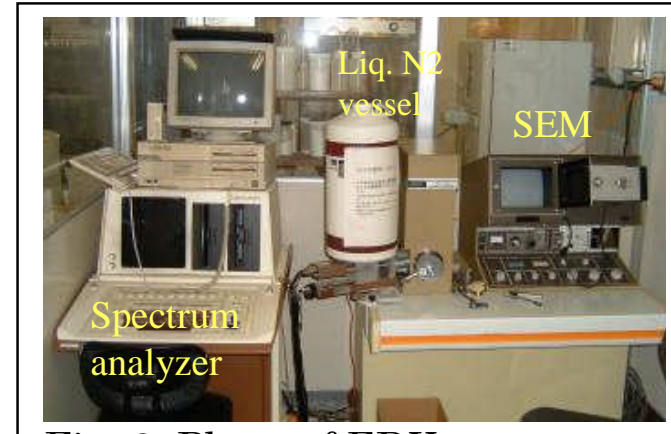

Fig. 8: Photo of EDX

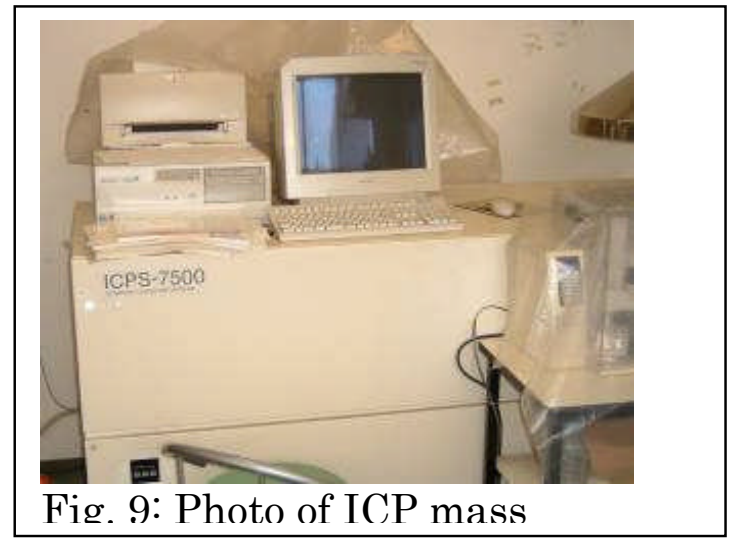




\section{Results}

We changed the input voltage stepwise as shown in Fig. 10. In this example, plasma formed at $120 \mathrm{~V}$. Once plasma formed, input current suddenly dropped. Meanwhile, the solution temperature reached roughly $80^{\circ} \mathrm{C}$. We increased voltage in stages to $350 \mathrm{~V}$ and then decreased it to $100 \mathrm{~V}$. Plasma continued at $100 \mathrm{~V}$ and ceased at $80 \mathrm{~V}$.

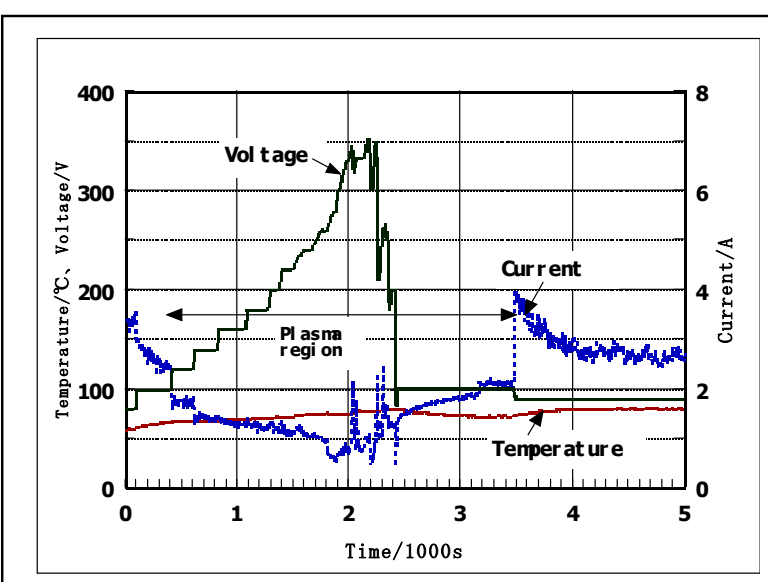

Fig. 10: Change for input voltage, current and Temperature

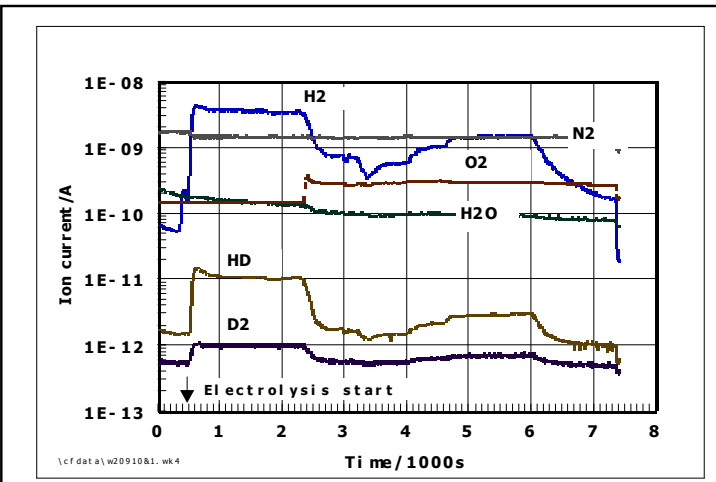

Fig. 11: Changes of the ion current of various masses

The gas collected by the funnel over the cathode is collected by the Q-mass-spectrometer continuously as shown in Fig. 11. It is mainly composed of three gasses: hydrogen, oxygen and nitrogen were always detected. Other gases were under the background levels and were not detected after electrolysis started. Oxygen gas increased after plasma electrolysis occurred. Hydrogen gas appeared when electrolysis started. When plasma electrolysis began, hydrogen production decreased while at the same time oxygen increased. The increased oxygen gas means that direct water decomposition by pyrolysis had begun. (Although total hydrogen production decreases when glow discharge begins, "excess hydrogen" increases. In other words, the ratio of hydrogen to input power sharply increases; glow discharge consumes much less power than ordinary electrolysis, and power falls even more than hydrogen production does.)

The behavior of the hydrogen isotope molecules is the same as hydrogen molecular ion.

Figure 12 shows changes over time for hydrogen and oxygen molecules and their ratio. The ratio increases as input voltage increases, reaching 0.45 at $2500 \mathrm{~s}$ and $350 \mathrm{~V}$. This means the gas from the cathode had begun direct decomposition by pyrolysis. The ratio of the hydrogen in gases released from the cathode is exactly same as the Faradaic value from the current. The equation is $\left(\mathrm{F}_{1}-0.116 \mathrm{I}\right)^{*} 0.667+0.116 \mathrm{I}$. Here, $\mathrm{F}_{1}$ is the rate of hydrogen gas estimated from the flow meter and, I is current. The factor of 0.116 is the rate of ordinal hydrogen generation, that is, $0.5 * 22414 / \mathrm{F}$ (F; Faraday constant of $96500 \mathrm{C} / \mathrm{mol}$ ). The factor of 0.667 is atomic ratio of hydrogen for water. 


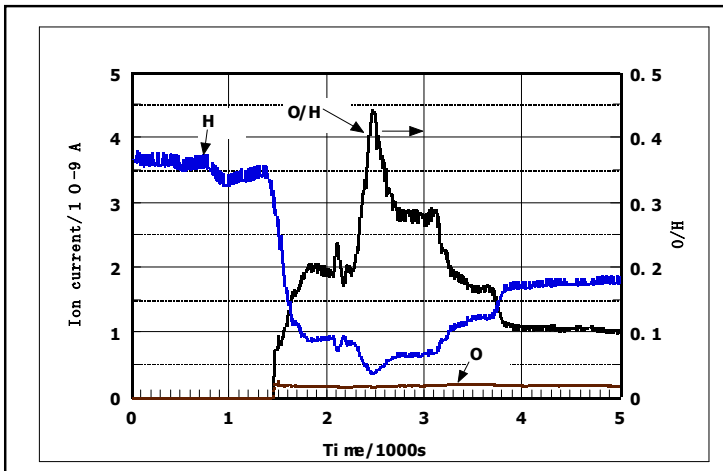

Fig. 12: $\mathrm{H} 2$ and $\mathrm{O} 2$ ion current and the ratio over time in $1000 \mathrm{~s}$.

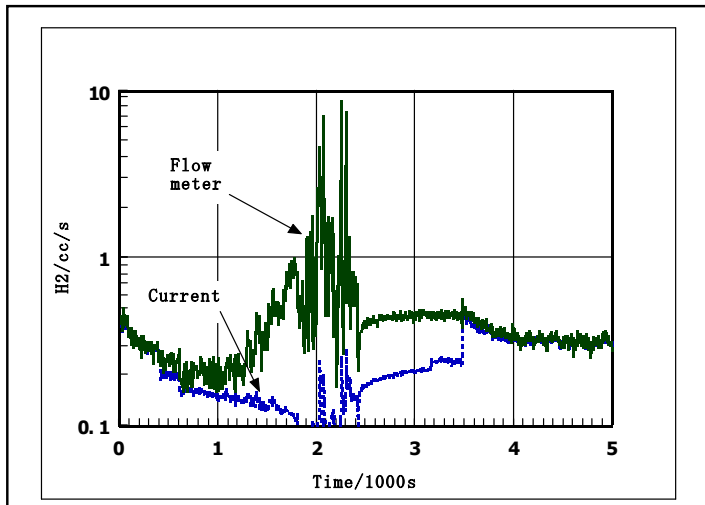

Fig. 13: Hydrogen generation over time.

Figure 13 shows the changes of hydrogen generation estimated from current, and measured with the gas flow meter. These rates were same with ordinary electrolysis. However, the value measured by the flow meter shows an upward deviation compared the value estimated from the current. The change of the ratio between these two values, i.e., current efficiency $(\varepsilon)$ and the ratio of the oxygen gas to the total generation with hydrogen from the cathode are shown in Fig. 4C. Here, $\varepsilon$ exceeds unity when the plasma electrolysis started; gas generation increased with the input voltage. It reached as $8000 \%$ at $350 \mathrm{~V}$ input voltage. The ratio of oxygen reached $30 \%$. This means that almost all of the hydrogen came from water decomposition (pyrolysis) during high voltage plasma electrolysis.

Figure 14 shows the change of the ratio between these two values, i.e., current efficiency $(\varepsilon)$ and the ratio of the oxygen gas to the total generation with hydrogen from the cathode. Here, the $\varepsilon$ exceeded unity when the plasma electrolysis started; gas generation increased a great deal with the input voltage. It reached as much as $8000 \%$ at $350 \mathrm{~V}$. For the entire run, the theoretical value of hydrogen generation calculated from the input current was $1144 \mathrm{cc}$, and the value measured during plasma electrolysis was $2190 \mathrm{cc}$. That is, the generation of excess hydrogen during a whole electrolysis reached $1046 \mathrm{cc}$. For measurements made only during times when the plasma was present, the measured value was $1470 \mathrm{cc}$ compared to the theoretical value were $460 \mathrm{cc}$, so the excess was $1010 \mathrm{cc}$. 
Figure 15 shows the $\varepsilon$ and $\mathrm{V}$ relationship. Here, it can be seen that $\varepsilon$ has a tendency of increase with input voltage. Some points of $\varepsilon$ value under $100 \mathrm{~V}$ in the figure show up to twice the theoretical value of unity; these points were obtained during plasma electrolysis. On the other hand, $\varepsilon$ remains at unity during normal electrolysis. It can be expected that if the input voltage is increased up to several hundred volts, then $\varepsilon$ would far exceed unity.
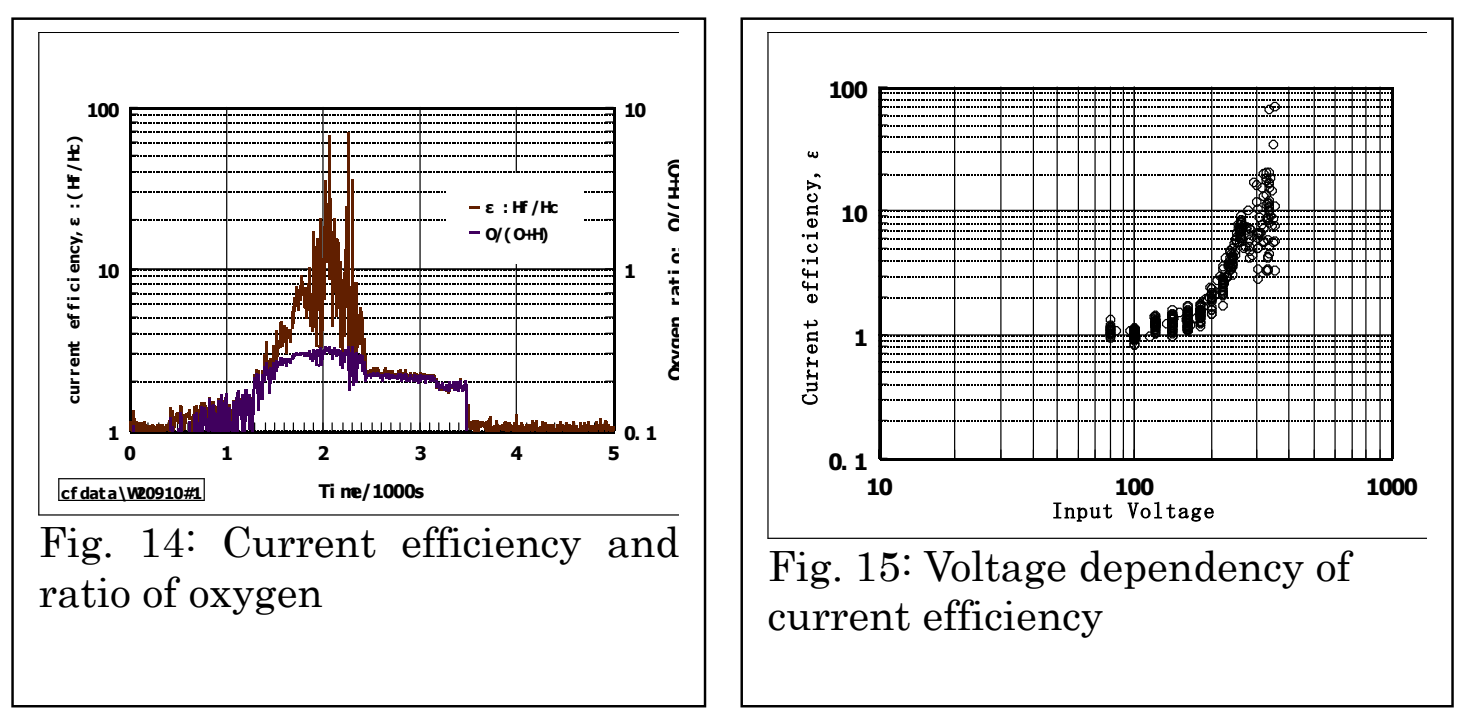

Figure 16 also shows the ratio of excess hydrogen to input power. These values were subtracted from the faradic hydrogen from the previous graph and converted into units of energy. It shows steep increase with the input voltage. It exceeds $10 \%$ and sometimes reaches $30 \%$.
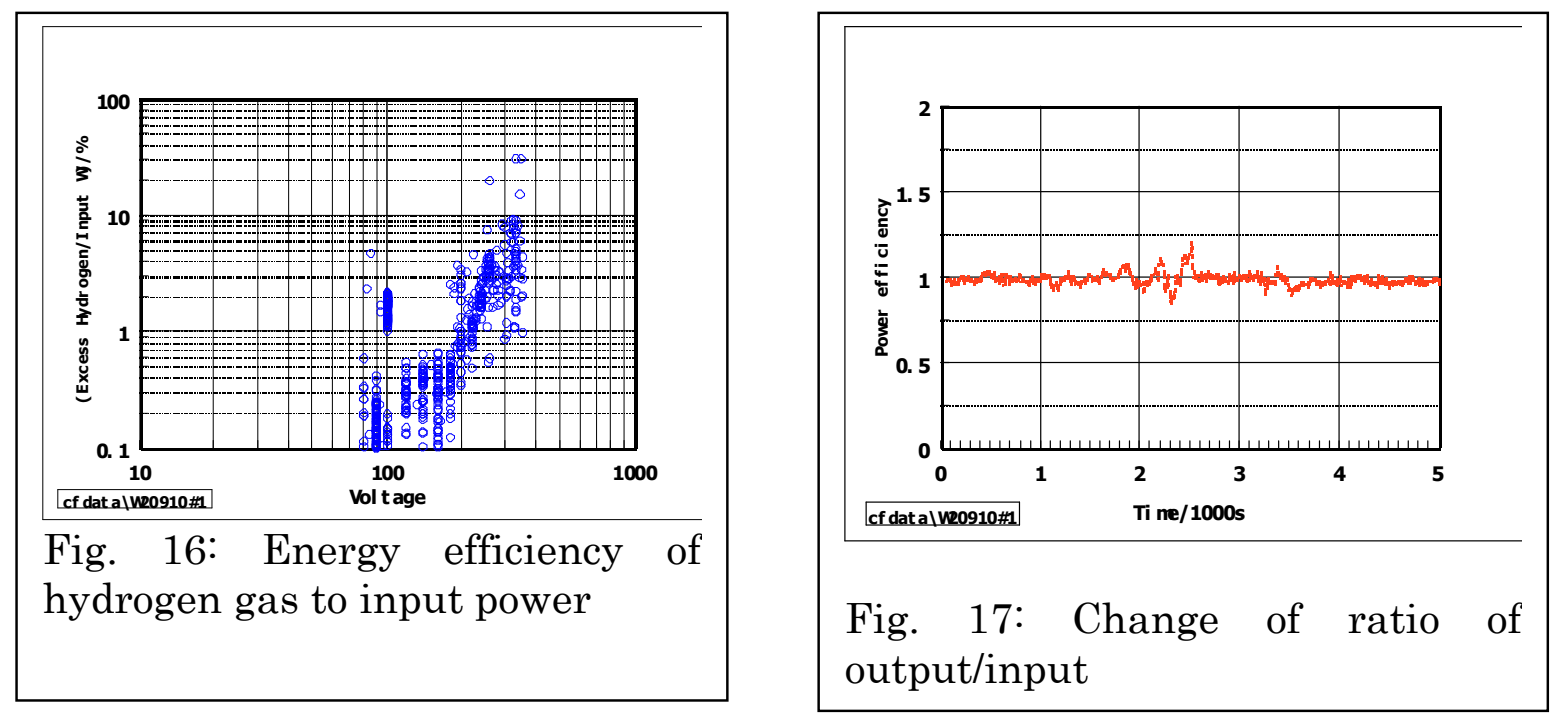

Figure 17 shows the heat balance for the run shown in Fig. 16. This indicates no excess heat. However, during this run, enthalpy release from excess hydrogen and oxygen evolution was not measured. So, there may be excess enthalpy, but in this case it can only be said that the heat balance was almost $100 \%$.

Figure 18 apparently indicates a tendency of the excess hydrogen generation with the 
negative excess heat. The total endothermic heat was calculated as $6540 \mathrm{~J}$ during plasma electrolysis.

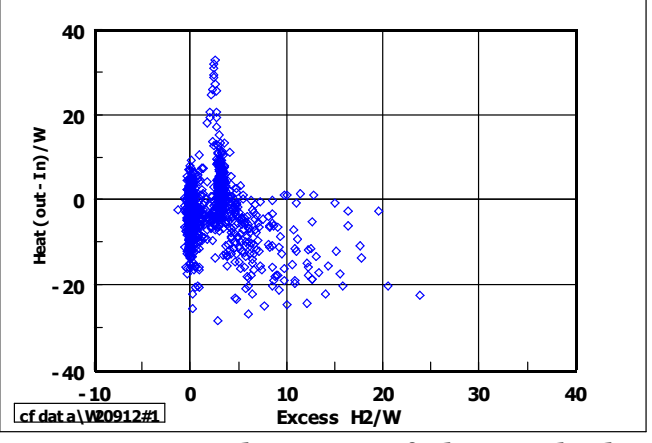

Fig. 18. Relation of heat balance and excess $\mathrm{H} 2$

Figure 18 shows an example in which no excess heat was generated. We changed the input voltage stepwise as shown in Fig. 19 . We used $0.1 \mathrm{M}$ of $\mathrm{K}_{2} \mathrm{CO}_{3}$ electrolyte. Plasma formed at $120 \mathrm{~V}$. Once plasma formed, input current dropped. The solution temperature reached $70^{\circ} \mathrm{C}$. We increased voltage in stages to $320 \mathrm{~V}$ and then decreased it to $100 \mathrm{~V}$. Plasma continued at $140 \mathrm{~V}$ and ceased at $100 \mathrm{~V}$.

Figure 20 shows the changes of hydrogen generation estimated from current and flow meter. The value estimated by flow meter exceeded the value of the current estimation.

If there were no excess heat, apparently the points for the excess hydrogen distribute around the no excess heat region like Fig. 21, that means the difference between heat out and electric power is zero.

Figure 22 shows the $\varepsilon$ and $\mathrm{V}$ relationship for plasma electrolysis in $0.1 \mathrm{M}$ of $\mathrm{K}_{2} \mathrm{CO}_{3}$ electrolyte.

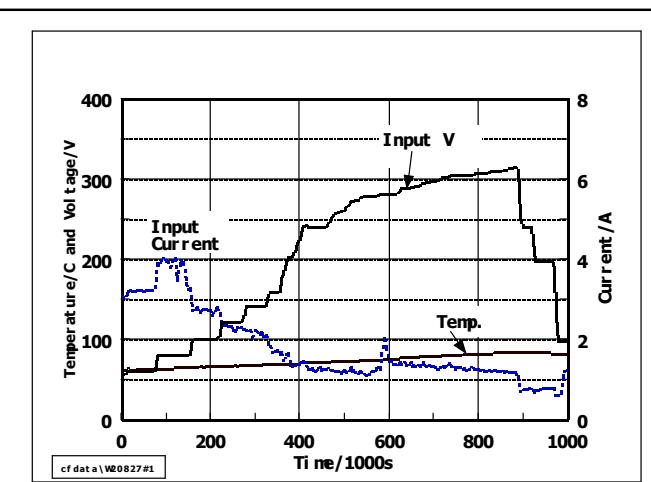

Fig. 19: Change for input voltage, current and Temperature

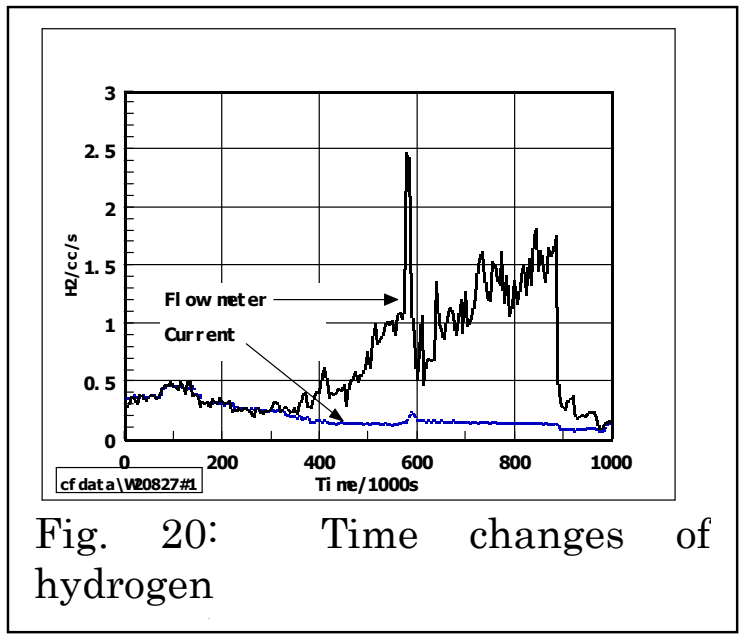



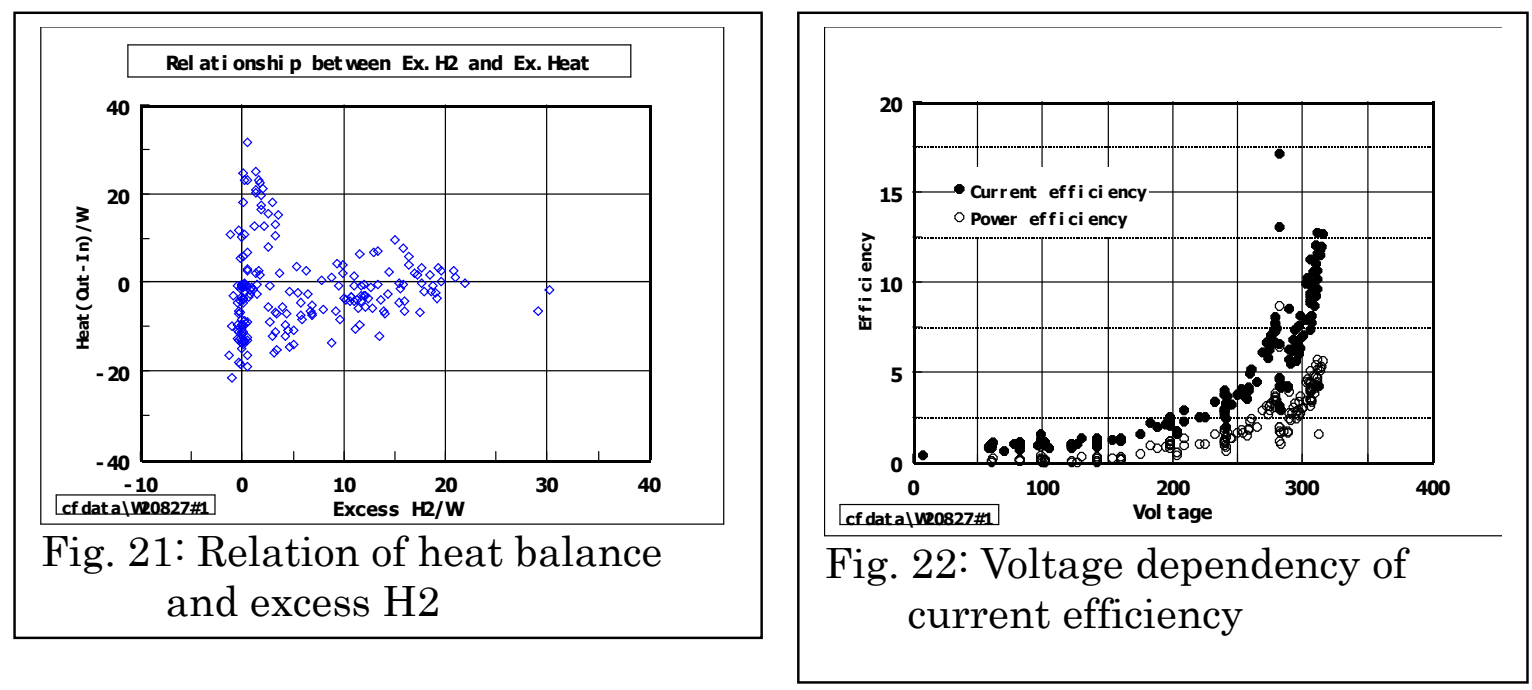

Figure 23 shows an example of excess heat. We changed the input voltage stepwise as shown in the figure. In this case, we used $0.05 \mathrm{M}$ concentrate $\mathrm{K}_{2} \mathrm{CO}_{3}$ electrolyte. The plasma electrolysis started at $120 \mathrm{~V}$. Once the plasma formed, input current dropped. The solution temperature was $80^{\circ} \mathrm{C}$. We increased the voltage stepwise to $320 \mathrm{~V}$ and then decreased it to $100 \mathrm{~V}$. Plasma continued at $160 \mathrm{~V}$ and ceased at $120 \mathrm{~V}$.

Figure 24 shows the changes of hydrogen generation estimated from current and flow meter. The value estimated by flow meter exceeded the value of the current estimation.

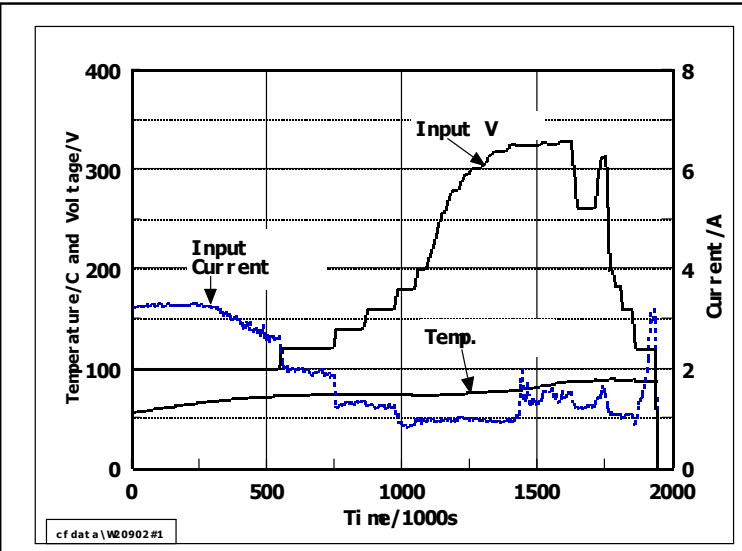

Fig. 23: Change for input voltage, current and Temperature.

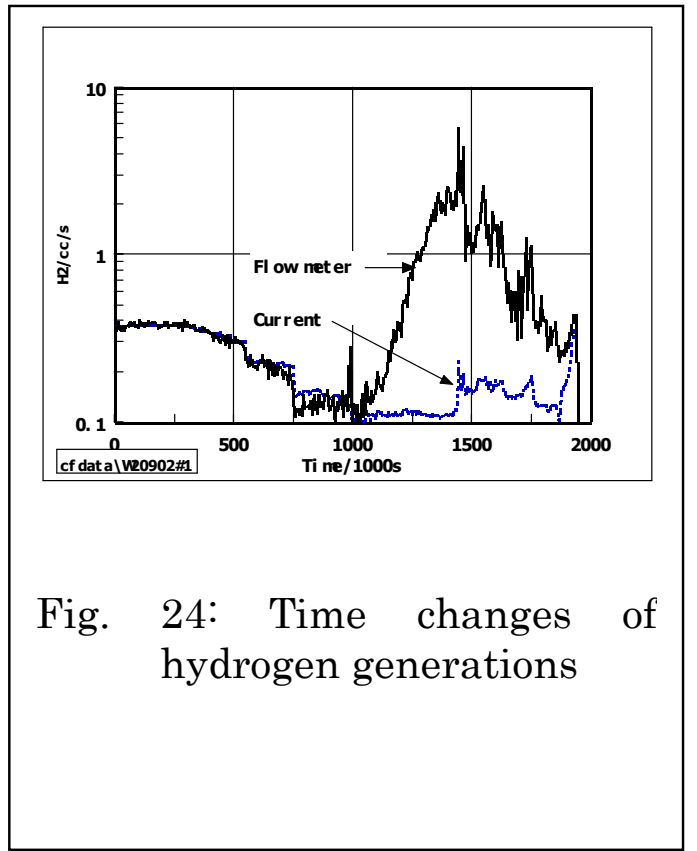



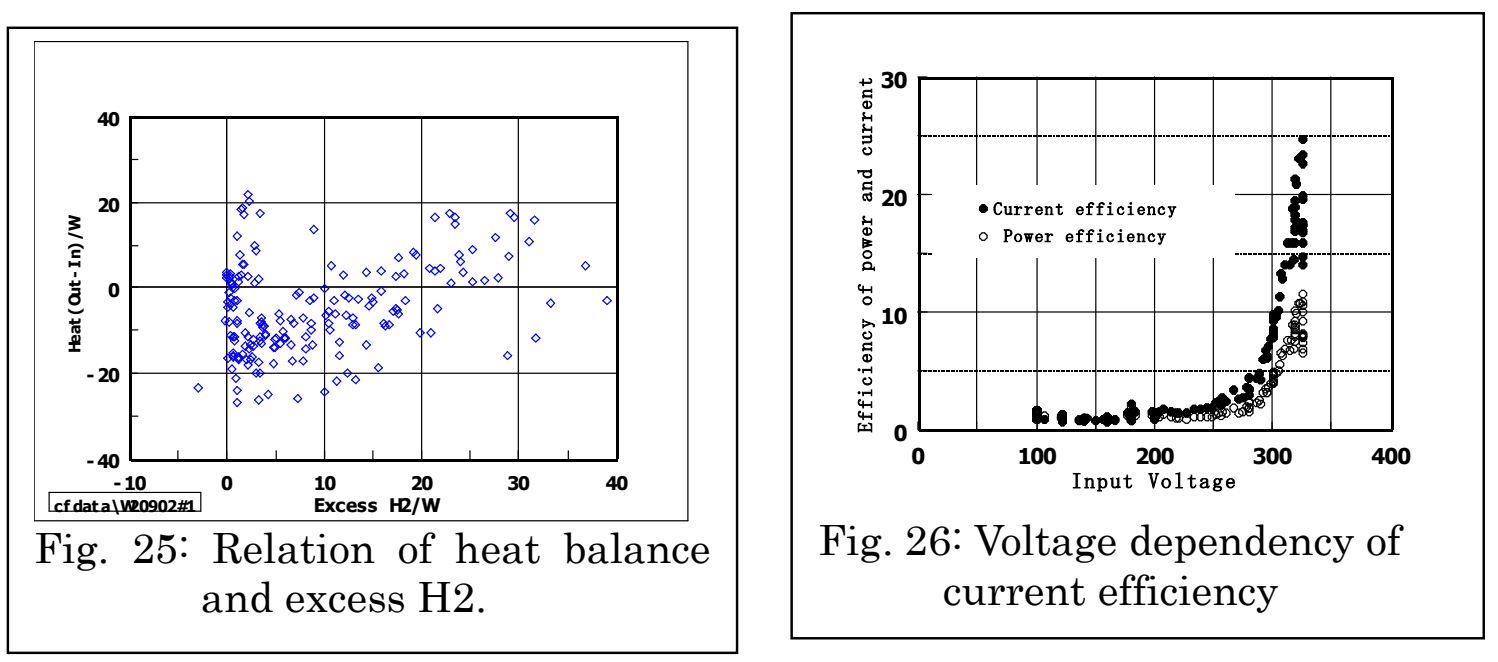

Figure 25 shows another relationship, between excess hydrogen and excess heat. In this case, the exothermic heat was estimated as $1100 \mathrm{~J}$ during plasma electrolysis. In all three cases, I have considered the contribution of excess hydrogen formation. However, apparently, the heat balance was not unity, it was changed by other parameters.

Figure 26 shows the $\varepsilon$ and $\mathrm{V}$ relationship for the plasma electrolysis in $0.05 \mathrm{M}$ of $\mathrm{K}_{2} \mathrm{CO}_{3}$ electrolyte.

We have analyzed the elements in the electrode and electrolyte by EDX and XPS, to estimate all of the elements in the electrolysis system. After electrolysis the element deposition for these three cases changed, as shown in Fig. 27. These depositions were also observed in various cases with electrolysis systems ${ }^{(8,9,10,11,12)}$.

There were several major elements observed in the system after excess energy was released. These were $\mathrm{Ca}, \mathrm{Fe}$ and $\mathrm{Zn}$. On the other hand, In and Ge were detected after systems were endothermic (absorbing heat). However, no major changes in elements were detected in the system when no exothermic or endothermic heat phenomenon occurred.

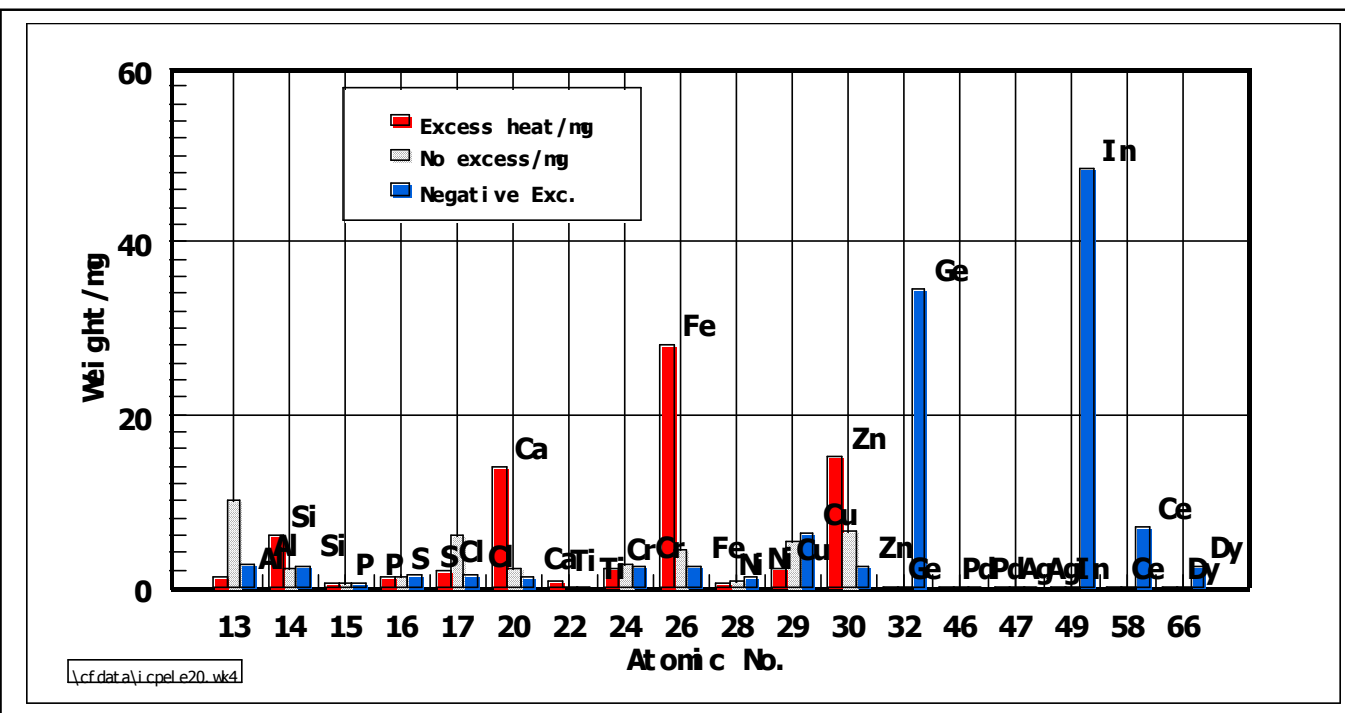

Fig. 27. Difference of element distributions after plasma electrolysis 


\section{Results}

It is still difficult to determine the conditions needed for excess energy generation. However, our results strongly suggest that we have to consider the contribution of the excess hydrogen. Sometimes the occupation percentage of excess hydrogen to the input energy reached to $30 \%$. After that, we obtained excess energy during the plasma electrolysis. However, excess energy generation only continued for several hundred seconds. One of the key factors that produce the excess energy seems the input voltage. Hydrogen generation and heat generation seems to correlate with the input voltage. Energy output can go over unity during plasma electrolysis if the input voltage is kept very high. Apparently, we have sometimes replicated excess heat generation at high input voltage region, even though the duration of the reaction is brief. One point of $\varepsilon$ value in the figure shows up to 80 times of the theoretical value of unity; the point was obtained during plasma electrolysis at $350 \mathrm{~V}$. On the other hand, $\varepsilon$ remains at unity for all other normal electrolysis. Our equipment is only capable of producing $350 \mathrm{~V}$. It can be expected that if the input voltage were increased to several hundred volts, the excess heat would greatly exceed unity.

\section{Conclusions}

We have reached several conclusions:

1. Current efficiency for the plasma electrolysis reaches $8000 \%$ to the input current.

2. Power efficiency for the plasma electrolysis reaches $30 \%$ to the input voltage.

3. In some cases, excess heat was observed.

4. In other cases, no and endothermic heat were confirmed.

5. The reaction products after electrolysis were changed with the excess heat generation.

\section{Acknowledgement}

We wish to express our thanks to the Thermal and Electric Technology Foundation for funding this research.

\section{References:}

(1) Arakawa, H., "Creation of new energy by photocatalyst: Technology of clean hydrogen fuel production from solar light and water", Reza Kenkyu Vol.25 (6), (1997) 425-430.

(2) Richard Rocheleau, Anupam Misra, Eric Miller, "Photo electrochemical hydrogen production", Proc., 1998 US-DOE, Hydrogen Program Review, NREL/CP-570-25315.

(3) Tadahiko Mizuno, Tadayoshi Ohmori, Tadashi Akimoto, Akito Takahashi, "Production of Heat during Plasma Electrolysis in Liquid", Jpn J. Appl. Phys., Vol.39, No.10 (2000) 6055-6061.

(4) T. Mizuno, T. Akimoto and T. Ohmori, "Confirmation of Anomalous Hydrogen Generation by Plasma Electrolysis", Proc. 4th meeting JCF research Soc., ed. H. Yamada, Oct., 17-18 (2002). Iwate Univ., Japan. (2002) 27-31

(5) A. Hickling and M. D. Ingram, Trans. Faraday Soc., Vol.60 (1964) 783

(6) A. Hickling, "Electrochemical processes in glow discharge at the gas-solution Interface" , Modern Aspects of Electrochemistry No.6, ed. by J. O’M. Bockris and B. E. Conway, Plenum Press New York (1971) 329-373

(7) S. K. Sengupta and O. P. Singh and A. K. Srivastava, J. Electrochem. Soc., Vol.145 (1998) 
(8) Tadahiko Mizuno, Tadashi Akimoto, Tadayoshi Ohmori, Akito Takahashi, Hiroshi Yamada and Hiroo Numata, "Neutron Evolution from a Palladium Electrode by Alternate Absorption Treatment of Deuterium and Hydrogen", Jpn J. Appl. Phys, Part 2, No.9A/B, Vol.40 (2001) L989-L991.

(9) Tadahiko Mizuno, Tadayoshi Ohmori and Michio Enyo, “Anomalous Isotopic Distribution in Palladium Cathode After Electrolysis”, J. New Energy, 1996. 1(2), p. 37.

(10) Tadahiko Mizuno, Tadayoshi Ohmori, Kazuya Kurokawa, Tadashi Akimoto, Masatoshi Kitaichi, Koichi Inoda, Kazuhisa Azumi. Sigezo Shimokawa and Michio Enyo, "Anomalous isotopic distribution of elements deposited on palladium induced by cathodic electrolysis", Denki Kagaku oyobi Kogyo Butsuri Kagaku, 1996. 64, p. 1160 (in Japanese).

(11) Tadahiko Mizuno, Tadayoshi Ohmori and Michio Enyo, "Isotopic changes of the reaction products induced by cathodic electrolysis in Pd", J. New Energy, 1996. 1(3), p. 31.

(12) Tadahiko Mizuno, Tadayoshi Ohmori and Michio Enyo, "Confirmation of the changes of isotopic distribution for the elements on palladium cathode after strong electrolysis in D2O solutions”, Int. J. Soc. Mat. Eng. Resources, 1998. 6(1), p. 45. 